\title{
Surfactant phospholipid composition of gastric aspirate samples differs between male and female very preterm infants
}

\author{
Foula Sozo ${ }^{1}$, Noreen Ishak ${ }^{1}$, Risha Bhatia ${ }^{2}$, Peter G. Davis ${ }^{2}$ and Richard Harding ${ }^{1}$
}

BACKGROUND: Among preterm infants, males have a greater incidence of respiratory distress and death than do females born at the same gestational age, likely due to sexrelated differences in lung maturation. Our aim was to determine whether surfactant phospholipid composition differs between male and female preterm infants.

METHODS: Gastric aspirate samples from male and female infants born between 25 and 30 weeks of gestation at The Royal Women's Hospital, Melbourne, Australia, were collected within $1 \mathrm{~h}$ after birth. Phospholipid composition was analyzed by electrospray ionization tandem mass spectrometry.

RESULTS: Preterm males had higher proportions of total phosphatidylinositol (PI) and phosphatidylserine 36:2, lower proportions of total sphingomyelin (S) and S 33:1 and 35:1, and a greater phosphatidylcholine $(\mathrm{PC}) / \mathrm{S}$ ratio than did females. The proportions of PC 30:0, PC 34:0, PC 34:2, PC 36:2, PC 36:3, and PC $38: 2$ differed between the sexes at different gestational weeks of birth; the proportion of PC 32:0 (dipalmitoylphosphatidylcholine) in males was lower than that in females at 25 weeks of gestation but higher at 27 weeks.

CONCLUSION: Phospholipid composition in pulmonary surfactant is different between male and female preterm infants of the same gestational age, which may contribute to the increased risk for respiratory morbidities in one sex.

I: is well established that, following preterm birth, male infants have poorer neonatal outcomes and a greater incidence of death than do females of the same gestational age (1-4). Male preterm infants require more initial respiratory and circulatory support than do females of the same gestational age, take longer to achieve targeted arterial oxygen saturation levels, have a greater oxygen dependency at 36 weeks' postmenstrual age, have longer hospital stays, have an increased incidence of RDS, BPD, and pulmonary hemorrhage, and have a greater requirement for postnatal corticosteroids (1,2,4-12). Evidence indicates that the "male disadvantage" in respiratory function is largely a result of lung immaturity relative to females. The respiratory "male disadvantage" following preterm birth continues throughout infancy, with preterm males exhibiting greater respiratory morbidity, including poorer lung function and an increased use of bronchodilators or inhaled steroids up to 24 months of age, independent of neonatal outcomes $(6,7,13)$.

Studies in humans and laboratory animals have demonstrated that maturation of the pulmonary surfactant system, including the secretion of surfactant during gestation, is delayed in males relative to females, and sex differences in the composition of pulmonary surfactant have been demonstrated in several species (14-19). Pulmonary surfactant comprises $~ 90 \%$ lipids, of which $90-95 \%$ are phospholipids. It contains seven major classes of phospholipids, with phosphatidylcholine (PC; also referred to as lecithin (L)) being the most abundant and the disaturated PC species dipalmitoylphosphatidylcholine (DPPC; also denoted as PC 16:0/16:0 or PC 32:0) having the greatest ability to reduce surface tension to near $0 \mathrm{mN} / \mathrm{m}$ under compression (20). As such, PCs are often used in indices of lung maturity. Based on surfactant composition (ratio of PC to sphingomyelin (S), the concentration and proportion of disaturated PC, and the proportion of phosphatidylglycerol (PG)), it has been suggested that, in the last 2 months of human gestation, lung maturation in male fetuses is retarded, relative to that in female fetuses, by 1.2-2.5 weeks $(16,17)$. Similarly, analyses of lung lavage and amniotic fluid from preterm fetal rabbits showed that females displayed an earlier increase in saturated PC/S and total PC/S ratios than did males (18).

Previous studies on sex differences in human lung maturation have focused on the major surfactant phospholipids, especially PC and its saturated species, primarily during late gestation; however, there is a paucity of data on differences in the composition of other surfactant phospholipids between males and females at earlier stages of fetal life, which could contribute to the male disadvantage in respiratory morbidity and mortality observed after very preterm birth. Therefore, our aim was to determine whether the composition of pulmonary phospholipids differs in newborn male and female preterm infants, and whether differences are related to gestational age. In order to analyze surfactant phospholipid composition, we have used gastric 
aspirates, samples of which have been used previously to assess surfactant maturity in preterm infants $(21,22)$. Gastric aspirate is likely to provide an index of lung maturity because, prior to the onset of feeding after birth, it primarily consists of ingested lung fluid and amniotic fluid, both of which are expected to contain pulmonary surfactant. We hypothesized that male infants born very preterm have altered surfactant phospholipid composition compared with females of the same gestational age at birth. We further hypothesized that sex differences in surfactant phospholipid composition differ with gestational age.

\section{METHODS}

\section{Sample Collection}

This study was approved by the Human Research Ethics Committee of The Royal Women's Hospital, Melbourne, Australia. Gastric aspirate samples were collected within $1 \mathrm{~h}$ after birth from male and female infants born at $<30$ weeks of gestation ( $25+0$ days $-29+6$ days $\left(25-29^{+6}\right)$ weeks of gestation; see Table 1$)$ at The Royal Women's Hospital between May 2010 and September 2012. Samples were collected from a maximum of 10 infants of each sex at each gestational week of birth within $1 \mathrm{~h}$ of birth; however, samples from only seven males and seven females could be obtained for the 25week group $\left(25-25^{+6}\right.$ weeks), and from five males in the 27 -week group $\left(27-27^{+6}\right.$ weeks; Table 1$)$. Samples from any infants with a congenital abnormality were excluded.

Infants recruited into this study were those who required respiratory support in the form of either intubation and ventilation or continuous positive airway pressure (CPAP) from birth; these infants routinely have a nasogastric or orogastric (NG/OG) feeding tube (5/6 Fr.) inserted soon after birth in order to vent air from the stomach following initial resuscitation. To ensure that the tip of the $\mathrm{NG} / \mathrm{OG}$ tube is in the stomach, fluid is aspirated $(0.5-1 \mathrm{ml})$ using a small syringe. The aspirated fluid is then tested on litmus paper to confirm its acidity and hence the correct positioning of the tube tip. For the present study, we took advantage of the fact that these infants would be routinely fitted with a NG/OG tube and have gastric samples aspirated. We obtained informed parental consent allowing us to keep the gastric aspirate samples for analysis, which otherwise would have been discarded. Samples were chilled and then stored at $-80^{\circ} \mathrm{C}$ until analysis.

\section{Surfactant Phospholipid Analysis}

Phospholipids in the gastric aspirate samples including the molecular species within the major phospholipid classes (lysophosphatidylcholine (LPC), PC, phosphatidylethanolamine (PE), PG, phosphatidylinositol (PI), phosphatidylserine (PS) and S) were isolated and then analyzed by electrospray ionization tandem mass spectrometry (PE Sciex API 4000 Q/TRAP; Framingham, MA) using a turbo-ion spray source and Analyst data system, as previously described (23). Briefly, phospholipids were extracted from gastric aspirate samples $(50 \mu \mathrm{l})$ with 2:1 chloroform-methanol $(1,000 \mu \mathrm{l})$ following the addition of internal standards (S 30:1, PC 13:0/13:0, LPC 13:0, PE 17:0/17:0, PS 17:0/17:0, and PG 17:0/17:0 (Avanti Polar Lipids, Alabaster, AL)). Liquid chromatographic separation was conducted on a $1.8 \mu \mathrm{m}$, $50 \times 2.1 \mathrm{~mm}$ C18 column (Zorbax; Agilent Technologies, Santa Clara, CA) at $300 \mu \mathrm{l} / \mathrm{min}$ using gradient conditions previously described (23). Individual lipids were quantified using scheduled multiple reactions monitoring in positive-ion mode and their concentrations calculated by relating the peak area of the lipid species to the peak area of the corresponding internal standard (23). The concentrations of all individual lipid species within each class were added to calculate the total concentration of each class of phospholipid; the total concentrations of each class of phospholipid were then added to calculate the total phospholipid concentration. Each individual molecular species within each phospholipid class was expressed as a molar percentage of its respective phospholipid class. Each phospholipid class was expressed as a molar percentage of the total phospholipids measured. Molecular species are denoted as either $A: x / B: y$ or $A+B: x+y$, where $A$ and $B$ are the number of carbon atoms in the fatty acid chains esterified at the $s n-1$ and $s n-2$ positions, respectively, and $x$ and $y$ are the number of double bonds in the fatty acid chains.

\section{Statistical Analysis}

Demographic data are presented as mean (SD) or median (range) as appropriate. These data were compared using Fisher's exact test for categorical data, Student's $t$-test for continuous variables, and MannWhitney's $U$-test for non-parametric data. Phospholipid data are presented as mean \pm SEM. Data were evaluated for normality using a Shapiro-Wilk test, and data were transformed where appropriate. The phospholipid composition of the gastric aspirate samples collected from infants born between 25 and $29^{+6}$ weeks of gestation was analyzed using a two-way analysis of variance, with sex $\left(P_{S}\right)$ and age $\left(P_{\mathrm{A}}\right.$; i.e., gestational age at birth) as factors. If there was a significant effect of gestational age, a least significant difference post hoc test was conducted to determine differences in phospholipid composition between each gestational week at birth. If there was a significant interaction effect $\left(P_{\mathrm{S} \times \mathrm{A}}\right)$, an unpaired Student's $t$-test was conducted to compare the phospholipid composition between males and females at each gestational week of birth. Statistical significance was set at $P<0.05$.

\section{RESULTS}

\section{Demographic Data}

The demographic details of the preterm infants used in our study are shown in Table 1 . There were no statistically significant differences in any of the variables analyzed between male and female infants at each gestational week of birth.

\section{Phospholipid Classes}

No overall effects of sex, gestational age, or interactions between these factors were present in the proportions of LPC (Figure 1a), PC (Figure 1b), PE (Figure 1c), PG (Figure 1d), and PS (Figure 1f) relative to the total amount of phospholipids measured. An overall sex effect and interaction effect were observed in the proportions of PI (Figure 1e) and S (Figure 1g); males overall (i.e., all males as a group) had a higher proportion of PI (most notable at 26 and 27 weeks of gestation) and a lower proportion of $S$ (most notable at 27 weeks of gestation) than did females. In addition, the proportion of $\mathrm{S}$ in samples from males and females combined changed with gestational age at birth, with the proportion of $\mathrm{S}$ being higher at 25 weeks than at 26 and 28 weeks, and higher at 27 weeks compared with that at 28 weeks.

\section{Phospholipid Species}

LPC species. An effect of gestational age was observed for the proportions of LPC 16:0, 18:1, 18:2, and 20:4 (Figure 2a,b,c,d, respectively). There was a tendency for the proportions of LPC 14:0 $\left(P_{\mathrm{A}}=0.063\right)$ and $16: 1\left(P_{\mathrm{A}}=0.070\right)$ to change in males and females with gestational age; however, these were not statistically significant (Supplementary Table S1). No overall effects of sex, age, or their interaction were observed for the proportions of LPC 14:0, 16:1, and 18:0; the mean \pm SEM of the 


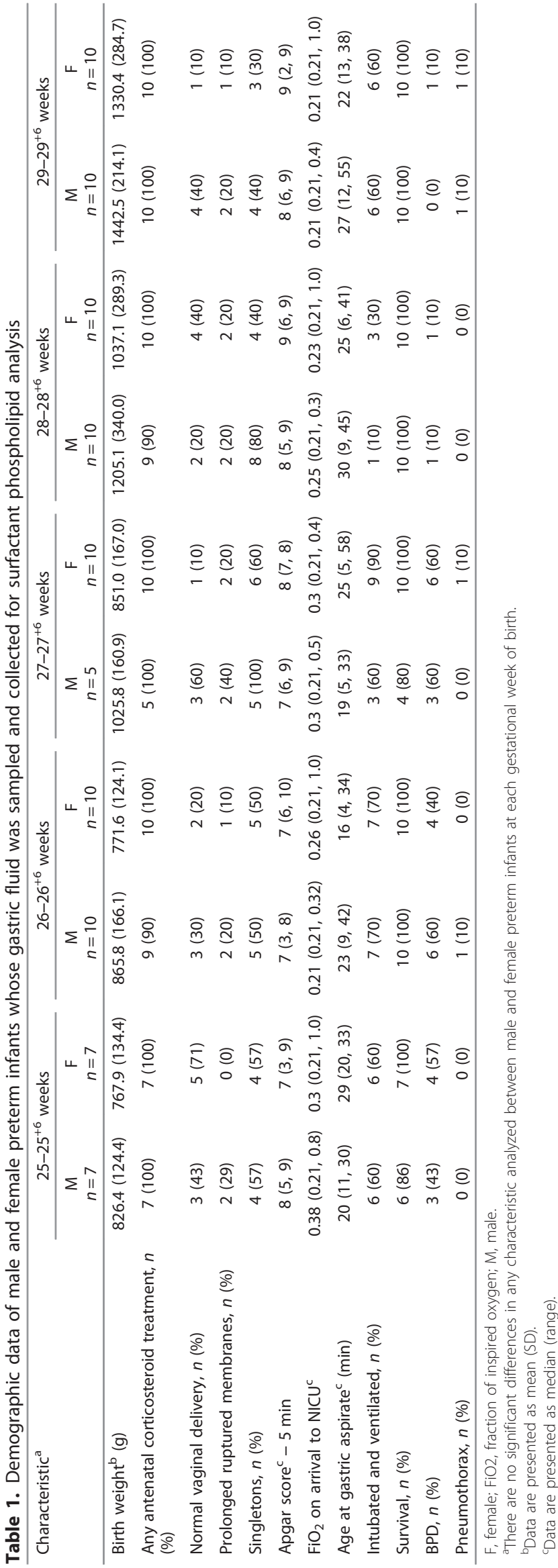

proportions of these species within LPC for each sex at each age is shown in Supplementary Table S1.

PC species. In both sexes combined, the proportion of PC 34:1 (Figure 3d) decreased with gestational age at birth, with the proportion being significantly lower at 28 and 29 weeks compared with that at 25 and 26 weeks. There was a significant age effect in the proportion of PC 32:0 (Figure 3b) such that values were higher at 28 and 29 weeks than at 25 and 26 weeks. In addition, the proportion of PC 32:0 was significantly lower in males than in females at 25 weeks, but it was significantly higher in males than in females at 27 weeks (Figure 3b). The proportions of PC 34:0 $\left(P_{\mathrm{A}}=0.079\right)$ and $38: 2\left(P_{\mathrm{A}}=0.059\right)$ tended to change with age when data from males and females were combined; these proportions were significantly different between males and females at specific gestational ages, with males at 27 weeks having a higher proportion of PC 34:0 (Figure 3c) and a lower proportion of PC 38:2 compared with females (Figure $3 \mathbf{h}$ ). An interaction effect was also observed for the proportions of PC 30:0, 34:2, 36:2, and 36:3 (Figure 3a,e,f, $\mathrm{g}$, respectively), where proportions of each lipid differed between males and females at specific gestational ages. No significant overall effects of sex or age or interaction effects were observed for the proportions of PC 32:1, 36:1, 38:4, 38:5, 16:0/20:4, and 16:0/22:6; the mean \pm SEM of the proportions of these species within PC is shown in the Supplemental Data (Supplementary Table S2).

PE species. There was a significant interaction effect in the proportion of PE 36:2 (Figure 4a), indicating that the effect of gestational age on the proportion of this species differed between males and females; there was a strong trend for males to have a lower proportion of PE 36:2 compared with females at 26 weeks of gestation $(P=0.051)$. There were no significant sex, age, or interaction effects in the proportions of the other PE species (34:1, 36:1, 36:3, 36:4, 38:3, 38:4, 38:5, 38:6, 40:4, and 40:6); however, the proportion of PE 34:2 $\left(P_{\mathrm{A}}=0.059\right)$ tended to change in males and females with gestational age at birth. The mean \pm SEM of the proportions of these species within PE is shown in Supplementary Table S3.

PG species. There were no overall effects of sex or age (or their interaction) in the proportions of PG 16:0/18:1 and 18:0/18:1, although there tended to be an interaction effect in the proportion of PG 18:1/18:1 $\quad\left(P_{S \times A}=0.064\right)$. The mean \pm SEM of the proportions of these species within PG is shown in Supplementary Table S4.

PI species. The proportions of PI 36:2 (Figure $4 \mathbf{b}$ ) and PI 36:3 (Figure 4c) in males and females increased with gestational age, with the proportions at 27 and 28 weeks being significantly higher than that at 25 weeks. There was an interaction effect in the proportion of PI 36:4, with males having a significantly higher proportion compared with females at 28 weeks (Figure $4 \mathbf{d}$ ). No overall sex, age, or interaction effects were observed for the proportions of PI 32:0, 32:1, 34:1, $36: 1,38: 3,38: 4$, and $40: 4$; the mean \pm SEM of the proportions of these species is shown in Supplementary Table S5. 

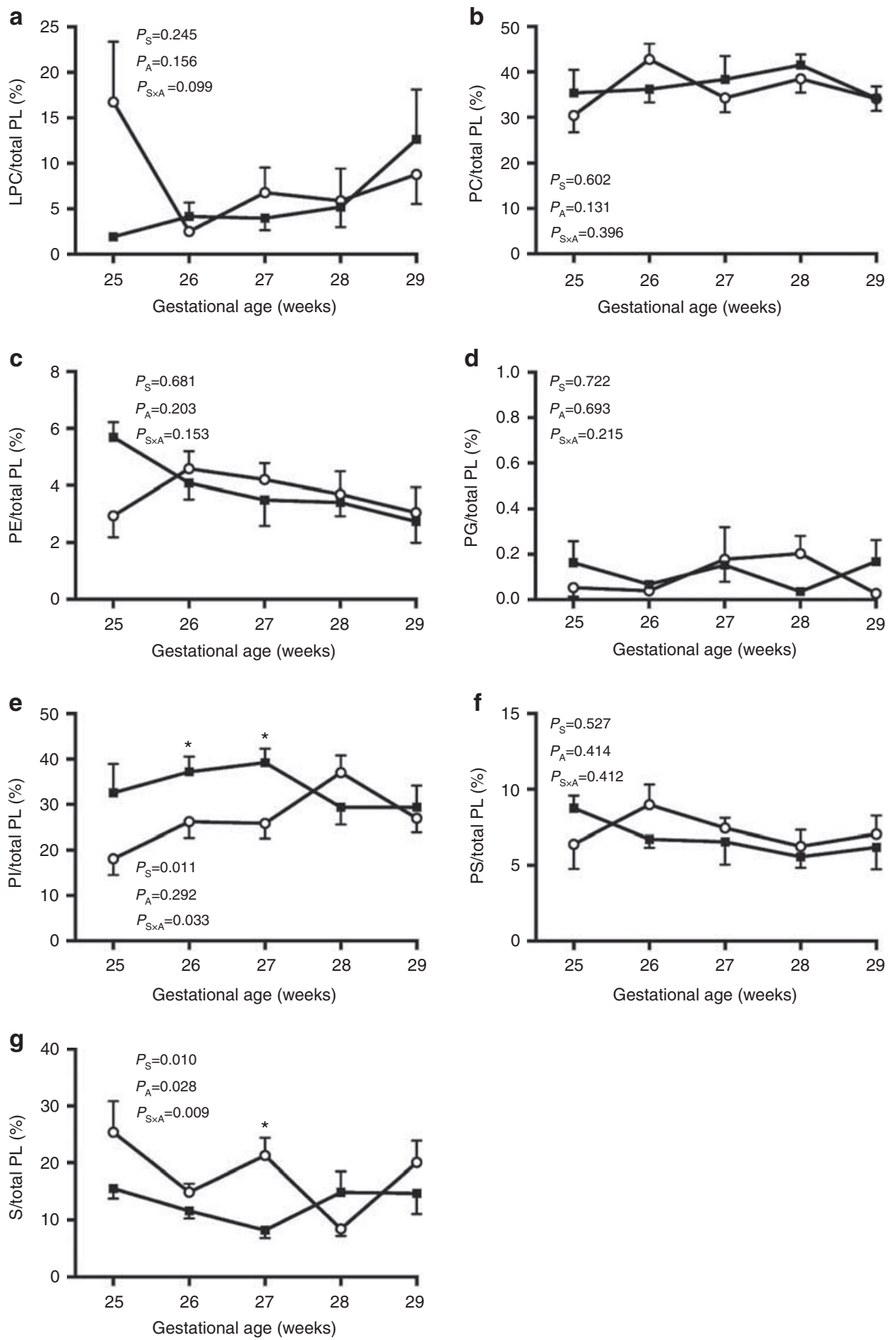

Figure 1. Class composition of phospholipids. (a) Proportion of phospholipid class lysophosphatidylcholine (LPC) in gastric aspirate samples from male (closed squares) and female (open circles) infants born at $25-29^{+6}$ weeks of gestation. (b) Proportion of phospholipid class phosphatidylcholine (PC) in gastric aspirate samples from male (closed squares) and female (open circles) infants born at $25-29^{+6}$ weeks of gestation. (c) Proportion of phospholipid class PE in gastric aspirate samples from male (closed squares) and female (open circles) infants born at $25-29^{+6}$ weeks of gestation. (d) Proportion of phospholipid class PG in gastric aspirate samples from male (closed squares) and female (open circles) infants born at $25-29^{+6}$ weeks of gestation. (e) Proportion of phospholipid class PI in gastric aspirate samples from male (closed squares) and female (open circles) infants born at $25-29^{+6}$ weeks of gestation. (f) Proportion of phospholipid class PS in gastric aspirate samples from male (closed squares) and female (open circles) infants born at $25-29^{+6}$ weeks of gestation. (g) Proportion of phospholipid class $S$ in gastric aspirate samples from male (closed squares) and female (open circles) infants born at 25 to $29^{+6}$ weeks of gestation. ${ }^{*} P<0.05$, male vs. female. 

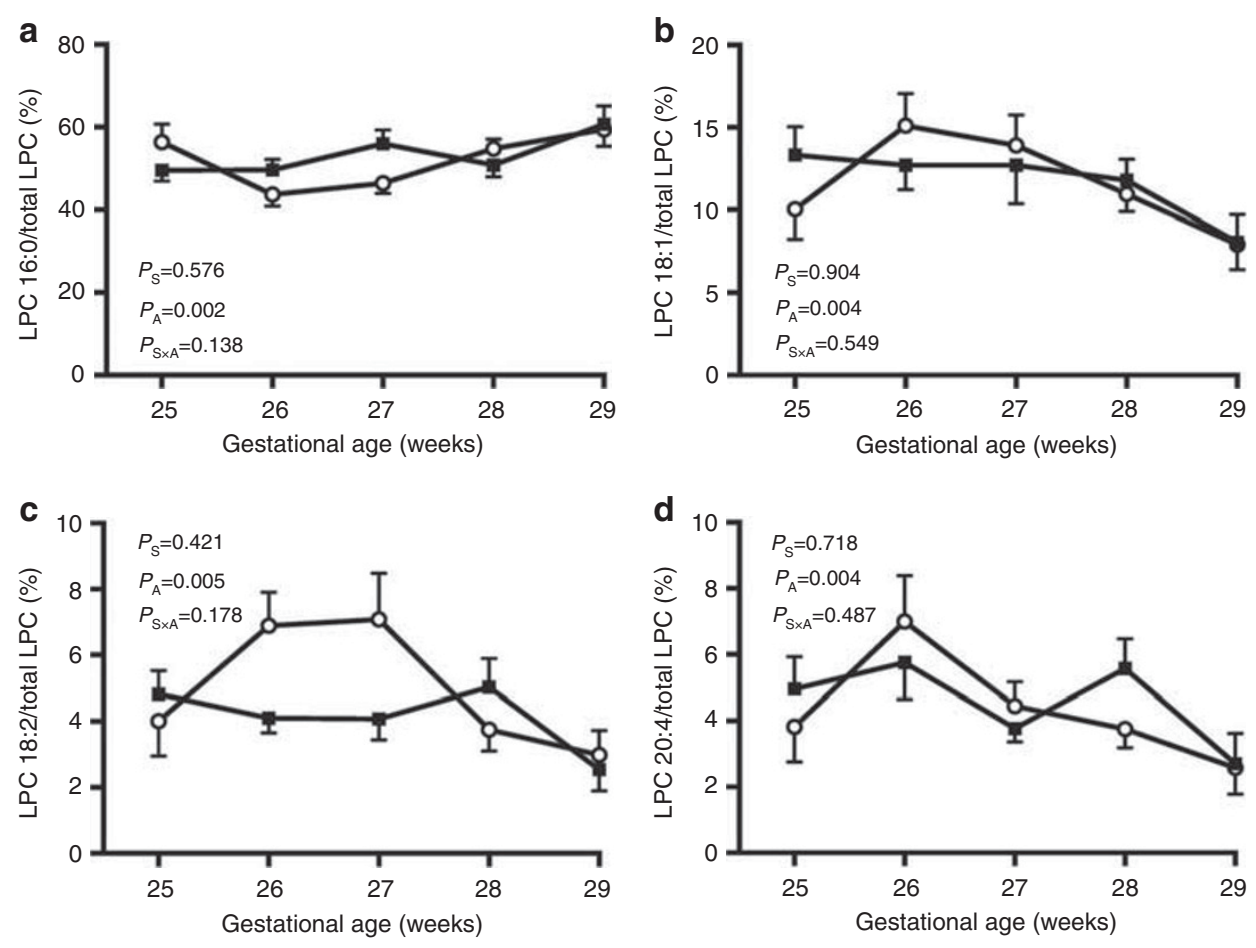

Figure 2. Proportions of the phospholipid molecular species of LPC. (a) Proportion of the phospholipid molecular species lysophosphatidylcholine (LPC) 16:0 in gastric aspirate samples from male (closed squares) and female (open circles) infants born at 25-29+6 weeks of gestation. (b) Proportion of the phospholipid molecular species LPC 18:1 in gastric aspirate samples from male (closed squares) and female (open circles) infants born at 25$29^{+6}$ weeks of gestation. (c) Proportion of the phospholipid molecular species LPC 18:2 in gastric aspirate samples from male (closed squares) and female (open circles) infants born at $25-29^{+6}$ weeks of gestation. (d) Proportion of the phospholipid molecular species LPC 20:4 in gastric aspirate samples from male (closed squares) and female (open circles) infants born at $25-29^{+6}$ weeks of gestation.

PS species. Males overall had a higher proportion of PS 36:2 than did females (Figure 4e). No overall sex, age, or interaction effects were observed for the proportions of PS $36: 1,38: 3,38: 4$, 40:5, and 40:6; the mean \pm SEM of these species is shown in Supplementary Table S6.

S species. Females overall had higher proportions of $\mathrm{S}$ 33:1 and S 35:1 than did males (Figure 5b,d, respectively). When data from both sexes were combined, the proportion of $S$ 33:1 (Figure 5b) decreased with increasing gestational age, with the proportion at 29 weeks being significantly lower than that at 25 , 26 , and 27 weeks. An interaction effect was observed in the proportions of S 32:1, 33:1, 34:2, 35:1, and 39:1 (Figure 5a,b,c, d,e, respectively), with males and females exhibiting different age-related changes in proportions. No overall sex, age, or interaction effects were observed in the proportions of the other S species (34:0, 34:1, 36:1, 38:1, 41:1, 41:2, and 42:1); the mean \pm SEM of the proportions of these species is shown in Supplementary Table S7.

\section{Phospholipid Maturity Ratios}

The ratio of PC/S was higher in the combined data from all males compared with that in the data from all females (Figure 6a). In the combined data from both sexes, PC/S increased at 27 weeks and 28 weeks compared with that at 25 weeks and 26 weeks, but decreased after 28 weeks
(Figure 6a). There was a tendency for PC/S to differ between the sexes at different gestational ages $\left(P_{\mathrm{S} \times \mathrm{A}}=0.064\right)$; however, this was not statistically significant (Figure 6a). No overall sex, age, or interaction effects were observed for PI/PG (Figure $6 \mathbf{b}$ ).

\section{DISCUSSION}

Previous studies have attributed the "male disadvantage" in preterm respiratory function to delayed lung development, in particular a delay in surfactant maturation and production $(16,17)$. Here we provide evidence showing that the phospholipid components of pulmonary surfactant differ according to gestational age and sex.

\section{Sex Differences in the PC/S Maturity Ratio}

Overall, preterm male infants had a greater PC/S ratio than did females, suggesting that males have greater lung surfactant maturity, although this tended to differ according to gestational age at birth. The data suggest that males born at 27 weeks of gestation have a higher PC/S ratio than do females, although this is likely due to a much higher ratio in one male infant compared with the other males at that age. Nonetheless, our data are in contrast to previous studies that have shown that males have a lower PC/S ratio than do females in late gestation. For example, amniotic fluid samples from human pregnancies with male fetuses have a lower PC/S 


\section{Articles | sozo et al.}

ratio than those with female fetuses from 30 to 40 weeks of gestation $(16,17)$. A similar sex difference in the PC/S ratio is observed in the amniotic fluid of fetal mice in late gestation
(24) and in lung lavage and amniotic fluid samples from fetal rabbits at $\sim 28$ weeks of human gestation equivalence (18). Compared with these previous studies, our study was
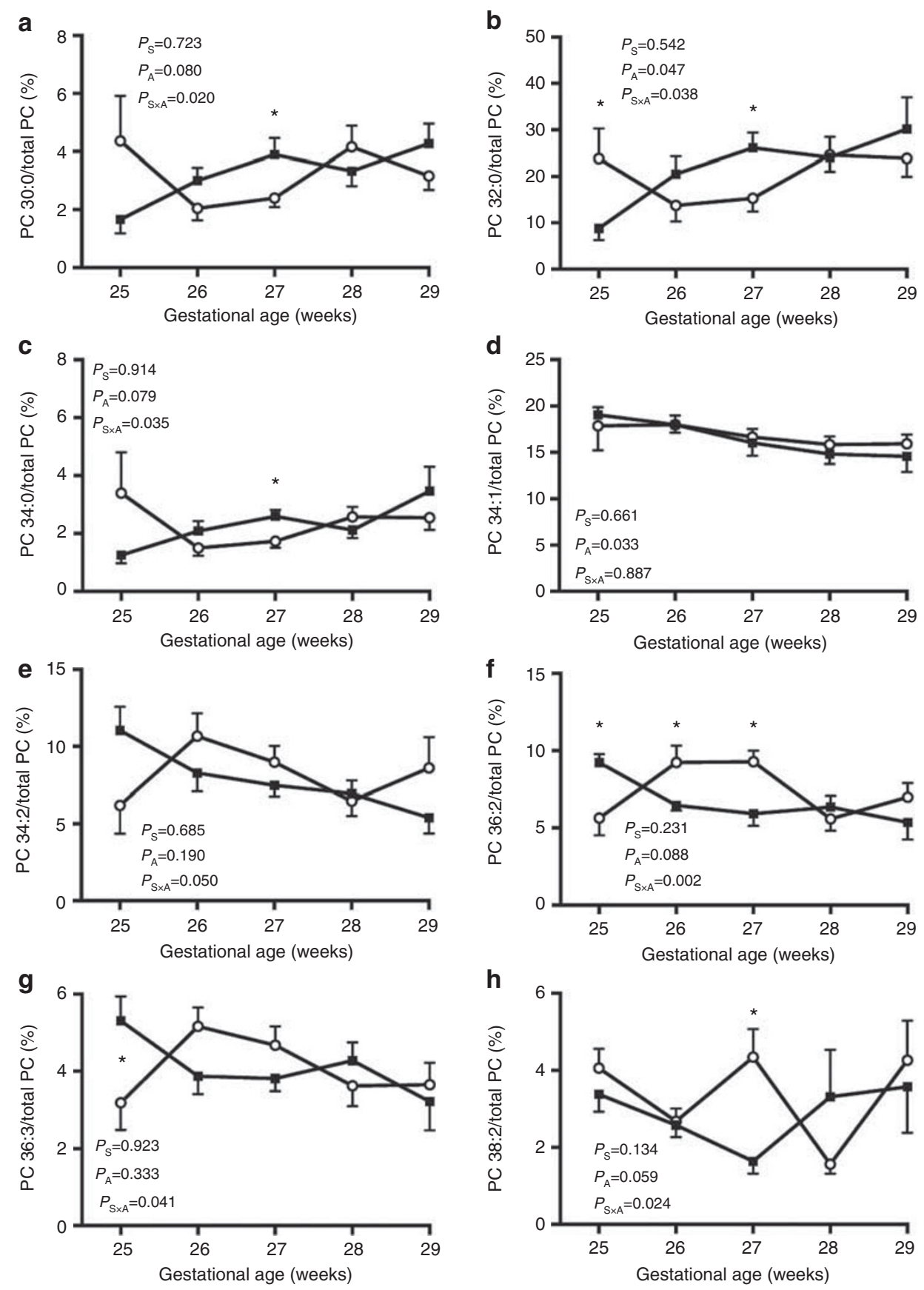

Figure 3. Proportions of the phospholipid molecular species of PC. (a) Proportion of the phospholipid molecular species phosphatidylcholine (PC) 30:0 in gastric aspirate samples from male (closed squares) and female (open circles) infants born at $25-29^{+6}$ weeks of gestation. (b) Proportion of the phospholipid molecular species PC 32:0 in gastric aspirate samples from male (closed squares) and female (open circles) infants born at 25-29 weeks of gestation. (c) Proportion of the phospholipid molecular species PC 34:0 in gastric aspirate samples from male (closed squares) and female (open circles) infants born at $25-29^{+6}$ weeks of gestation. (d) Proportion of the phospholipid molecular species PC $34: 1$ in gastric aspirate samples from male (closed squares) and female (open circles) infants born at $25-29^{+6}$ weeks of gestation. (e) Proportion of the phospholipid molecular species PC 34:2 in gastric aspirate samples from male (closed squares) and female (open circles) infants born at $25-29^{+6}$ weeks of gestation. (f) Proportion of the phospholipid molecular species PC $36: 2$ in gastric aspirate samples from male (closed squares) and female (open circles) infants born at $25-29^{+6}$ weeks of gestation. (g) Proportion of the phospholipid molecular species PC 36:3 in gastric aspirate samples from male (closed squares) and female (open circles) infants born at $25-29^{+6}$ weeks of gestation. (h) Proportion of the phospholipid molecular species PC $38: 2$ in gastric aspirate samples from male (closed squares) and female (open circles) infants born at $25-29^{+6}$ weeks of gestation. ${ }^{*} P<0.05$, male vs. female. 

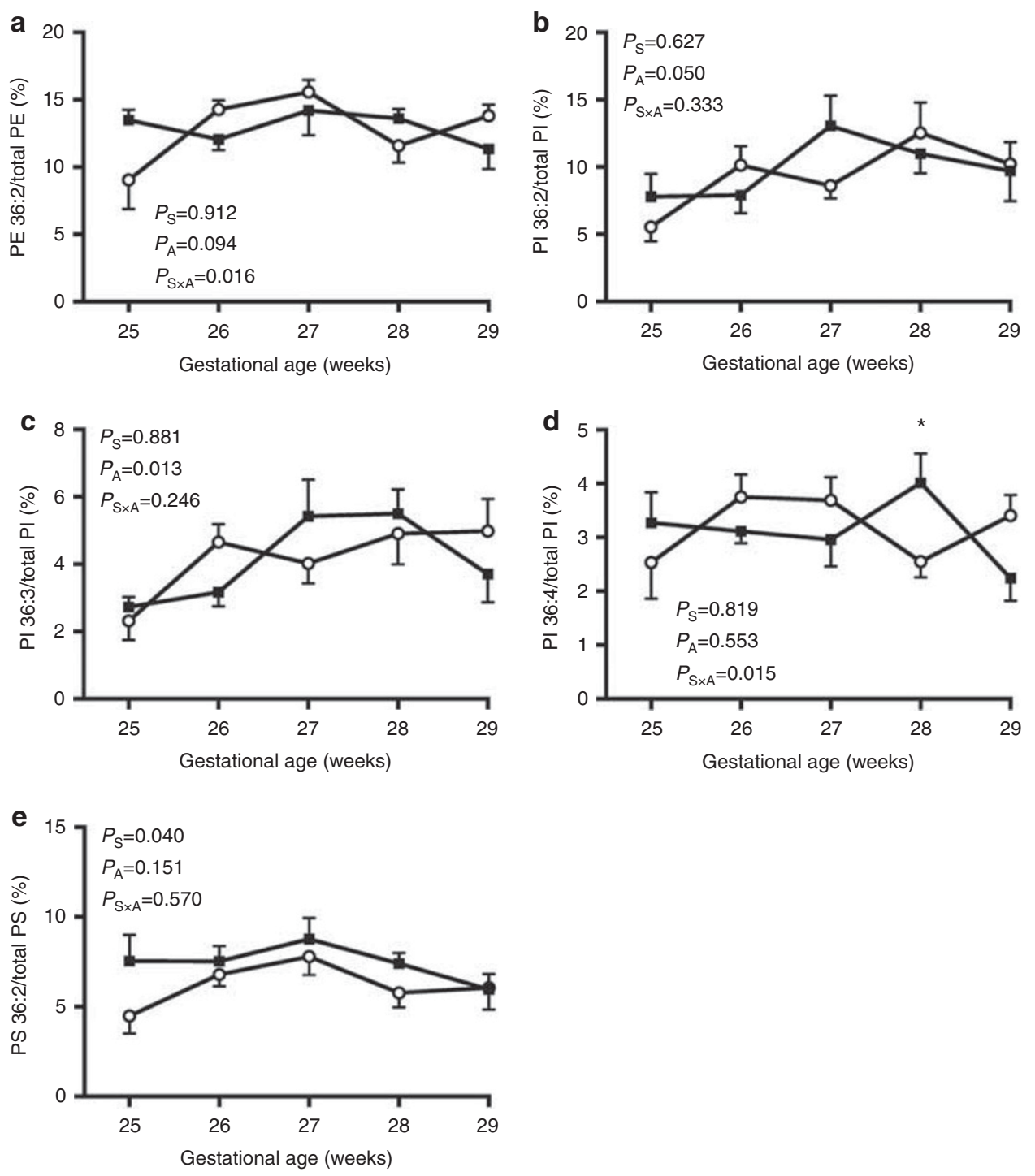

Figure 4. Proportions of the phospholipid molecular species of PE, PI and PS. (a) Proportion of the phospholipid molecular species PE 36:2 in gastric aspirate samples from male (closed squares) and female (open circles) infants born at $25-29^{+6}$ weeks of gestation. (b) Proportion of the phospholipid molecular species PI 36:2 in gastric aspirate samples from male (closed squares) and female (open circles) infants born at $25-29^{+6}$ weeks of gestation. (c) Proportion of the phospholipid molecular species PI 36:3 in gastric aspirate samples from male (closed squares) and female (open circles) infants born at $25-29^{+6}$ weeks of gestation. (d) Proportion of the phospholipid molecular species PI 36:4 in gastric aspirate samples from male (closed squares) and female (open circles) infants born at $25-29^{+6}$ weeks of gestation. (e) Proportion of the phospholipid molecular species PS $36: 2$ in gastric aspirate samples from male (closed squares) and female (open circles) infants born at $25-29^{+6}$ weeks of gestation. ${ }^{*} P<0.05$, male vs. female.

performed on gastric aspirate samples collected from infants soon after birth (rather than samples collected during pregnancy) and who were born at earlier gestational ages. Interestingly, in the bronchoalveolar lavage fluid of male lambs collected at $4 \mathrm{~h}$ after preterm birth there was a lower $\mathrm{PC} / \mathrm{PE}$ ratio (equivalent to the $\mathrm{PC} / \mathrm{S}$ ratio) than in females (19); however, there was no sex difference in the PC/S ratio in amniotic fluid (19) or in lung liquid (25) prior to preterm birth or in bronchoalveolar lavage fluid collected at $8 \mathrm{~h}$ after preterm birth in lambs managed on CPAP (26). In addition, no sex difference in the composition of surfactant is observed in very immature or mature rabbit fetuses (18), or in rhesus monkeys in the last trimester-equivalent (27), suggesting that the timing of sample collection, the species, and potentially the sample source influence the data.

\section{Sex Differences in the Composition of PC}

The most abundant phospholipid species within PC and the phospholipid with the greatest ability to reduce surface tension within the lungs in most mammals is the disaturated phospholipid DPPC (PC 32:0) (20). The proportion of PC 32:0 in the gastric aspirate of males was lower than that in females at 25 weeks of gestation but higher in males than in females at 27 weeks, suggesting that males born at 25 weeks 

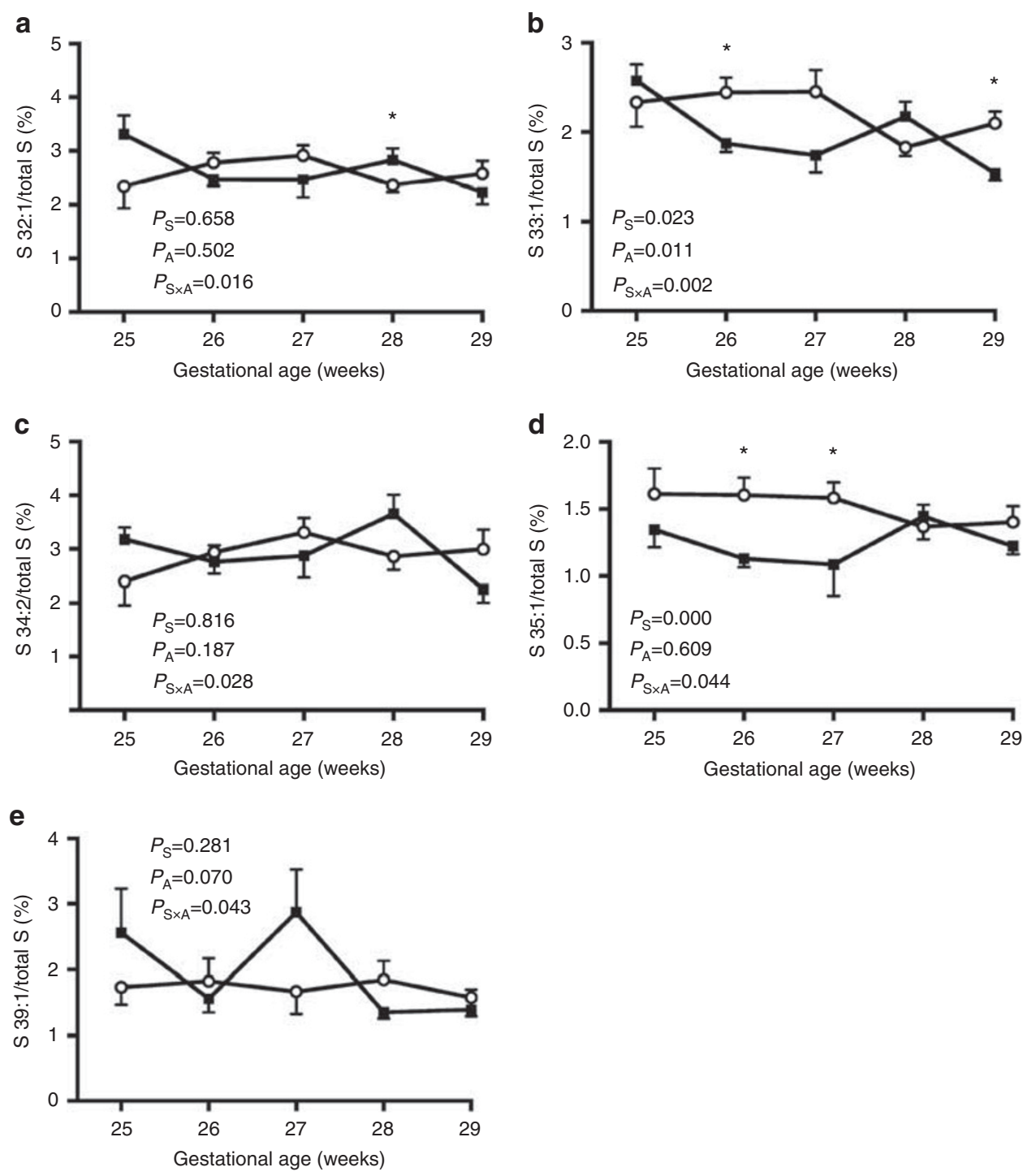

Figure 5. Proportions of the phospholipid molecular species of S. (a) Proportion of the phospholipid molecular species $S$ 32:1 in gastric aspirate samples from male (closed squares) and female (open circles) infants born at $25-29^{+6}$ weeks of gestation. (b) Proportion of the phospholipid molecular species S 33:1 in gastric aspirate samples from male (closed squares) and female (open circles) infants born at 25-29+6 weeks of gestation. (c) Proportion of the phospholipid molecular species S 34:2 in gastric aspirate samples from male (closed squares) and female (open circles) infants born at $25-29^{+6}$ weeks of gestation. (d) Proportion of the phospholipid molecular species S 35:1 in gastric aspirate samples from male (closed squares) and female (open circles) infants born at $25-29^{+6}$ weeks of gestation. (e) Proportion of the phospholipid molecular species $S 39: 1$ in gastric aspirate samples from male (closed squares) and female (open circles) infants born at $25-29^{+6}$ weeks of gestation. ${ }^{*} P<0.05$, male vs. female.

may be more vulnerable to respiratory distress than would females of the same age. Previous studies in humans have reported that the concentration of saturated PC and the proportion of disaturated PC are lower in amniotic fluid from pregnancies with males than with females between 30 and 40 weeks of gestation $(16,17)$. Based on the observed sex differences in the proportion of saturated PCs in the human fetus, it is likely that there may be windows of increased vulnerability for males compared with females in terms of their ability to reduce surface tension at the air-liquid interface.

Previous studies in different animal models have shown conflicting data with regard to sex differences in PC composition. As in humans, disaturated PC content in lategestation fetal rats is greater in the lungs of females than in males (14). In contrast, no sex difference in disaturated PC is observed in non-human primates in the last-trimester equivalent (27) or in fetal rabbits at an age that equates to $\sim 28$ weeks of human gestation (28). Similarly, there are no sex differences in PC composition in near-term mice (24), in sheep fetuses $(19,25)$, or in preterm lambs that are spontaneously breathing and managed on CPAP (26) or anesthetized and ventilated (29). Conversely, spontaneously breathing male preterm lambs not managed on CPAP have lower proportions of DPPC and PC than do females (19). These studies suggest that timing of sample collection is an 

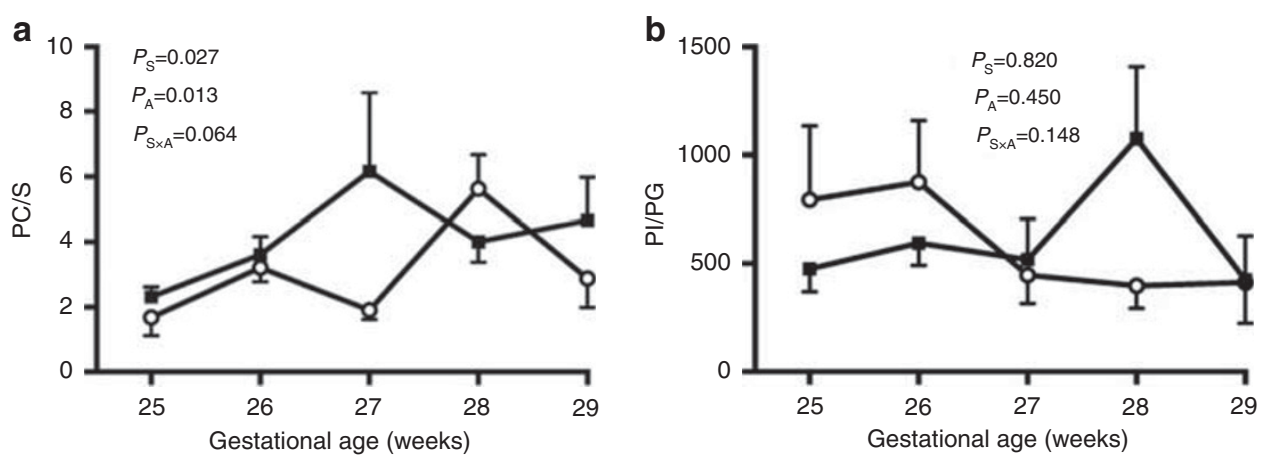

Figure 6. Phospholipid maturity ratios. (a) The phospholipid maturity ratio PC/S in gastric aspirate samples from male (closed squares) and female (open circles) infants born at $25-29^{+6}$ weeks of gestation. (b) The phospholipid maturity ratio PI/PG in gastric aspirate samples from male (closed squares) and female (open circles) infants born at $25-29^{+6}$ weeks of gestation.

important consideration when evaluating phospholipid composition.

The proportions of the saturated PCs 30:0 (PC 16:0/14:0; palmitoyl-myristoyl PC) and 34:0 (PC 16:0/18:0; palmitoylstearoyl PC) and the unsaturated PCs 34:2 (PC 16:0/18:2; palmitoyl-linoleoyl PC), 36:2 (PC 18:1/18:1; dioleoyl PC), $36: 3$, and $38: 2$ in gastric aspirate differed between preterm males and females at different gestational weeks of birth. Although the role of each of these individual PC species is unclear, it is known that saturated PCs help reduce surface tension at the air-liquid interface because of their molecular structure, namely their ability to be tightly packed (30). In contrast, unsaturated PCs are less able to reduce surface tension, although they are more fluid at body temperature; this characteristic enables greater surface film respreading and adsorption to the air-liquid interface (20). Therefore, alterations in PC composition can alter the surfactant properties. There are few data on sex differences in specific molecular species of PC (other than DPPC); however, a previous study in preterm lambs found higher proportions of PC 34:2 and PC 36:2 in the bronchoalveolar lavage fluid of males than in those of females at $4 \mathrm{~h}$ after delivery (19). In the present study, the proportion of PC 36:2 was higher in males born at 25 weeks of gestation compared with that in females; however, it was greater in females born at 26 and 27 weeks of gestation. PC $34: 2$ and PC 36:2 are considered to be plasma PCs, and an increase in plasma PCs may indicate a decreased ability for surfactant to lower surface tension in the lungs as plasma proteins are known to inhibit surfactant function (31). Overall, based on our data on PC composition, it is possible that males born at 25 weeks of gestation are more vulnerable than females to surfactant dysfunction, whereas females are more vulnerable at 27 weeks of gestation.

\section{Sex Differences in Acidic Phospholipids}

PG and PI are anionic phospholipids that are thought to be important in enhancing the surface activity of DPPC by promoting its adsorption to the air-liquid interface; PG is also stipulated to have other roles (30). It has previously been reported that PG is produced late in gestation $(16,27,28,32,33)$; thus, PG or the PI/PG ratio is often used as an indicator of lung maturity (32). In humans, PG reportedly first appears in amniotic fluid at 34 weeks of gestation in females and at 35 weeks in males, and the rate of increase in the proportion of PG is higher in females than in males (16). In the present study, there were no sex differences in the proportion of PG or in the PI/PG ratio; however, because of the earlier gestational ages examined, PG was detected at very low levels. Similarly, there is no apparent sex difference in the proportion of PG in sheep prior to preterm delivery or soon after preterm delivery, although the levels of PG are also low at this age $(19,25,26)$. In contrast to PG, the proportion of PI in amniotic fluid reportedly increases from 28 weeks of gestation in humans and then gradually decreases after 36 weeks (33), with female fetuses having levels lower than those of males after 37 weeks (16). In fetal rabbits, males tend to have a lower proportion of PI in lung lavage than females at 0.9 of term, but at term, male fetuses tend to have a higher proportion of PI and a lower proportion of PG than females (28). In contrast, there is no sex difference in the proportion of PI in sheep prior to or soon after preterm delivery $(19,25,26)$. In the present study, preterm males had a higher proportion of PI than did females, which was most apparent at 26 and 27 weeks of gestation. Since previous studies have indicated a rise in PI levels after 28 weeks of gestation (33), the higher proportion of PI in males at the earlier gestational ages examined in our study could be beneficial in reducing surface tension; however, experimental studies suggest that increasing the proportion of PI in surfactant has no significant effect on surfactant function (20).

\section{Sex Differences in the Composition of Other Phospholipids}

Preterm males had a lower proportion of total $S$ in their gastric aspirate than did females, which was most evident at 27 weeks of gestation; the lower proportion of $S$ contributes to the higher PC/S ratio observed in preterm males as there was no sex difference in the proportion of PC. Sphingomyelin is found in cellular membranes and is a minor component of surfactant itself; it is usually only used as a reference for the 
measurement of PC production rather than being perceived to have a role in surfactant function (32). However, there are studies in which an increase in the levels of "membrane phospholipids" has been associated with lung injury (20). PE is also considered to be a membrane phospholipid that is present in surfactant (20), although unlike sphingomyelin, no significant sex difference in PE proportion was observed in the present study. Compared with females, preterm males also had a higher proportion of PS 36:2 in gastric aspirate, a lower proportion of $S 33: 1$, and a lower proportion of S 35:1, which was most notable at 26 and 27 weeks of gestation. It is unknown whether these differences in phospholipid composition are likely to result in alterations in surfactant function as the specific roles of these phospholipid species are not clear.

\section{Changes in Phospholipid Composition with Age}

The proportions of numerous phospholipid species changed with increasing gestational age in both males and females: LPC 16:0, LPC 18:1, LPC 18:2, LPC 20:4, PC 32:0, PC 34:1, PI $36: 2$, PI $36: 3$, and $S 33: 1$. In addition, the proportion of $S$ and consequently the $\mathrm{PC} / \mathrm{S}$ ratio were altered by gestational age in male and female preterm infants. As expected, PC/S increased with advancing gestational age, indicating increasing lung maturity. The proportion of PC 32:0 was greater at 28 and 29 weeks than at 25 and 26 weeks of gestation, also suggesting an improvement in surfactant function (i.e., surface tension lowering ability) with advancing gestational age. In contrast, the proportion of PC 34:1 (PC 16:0/18:1; palmitoyl-oleoyl PC) decreased with increasing gestation despite being one of the major unsaturated PCs in surfactant. The proportions of each of the aforementioned LPC species were differentially altered by age; although the functions of these specific LPC species are unknown, it is known that LPC is associated with lung injury (34). The significance of changes in the proportions of PI 36:2, PI 36:3, and S 33:1 across different gestational ages at birth is unknown.

\section{Further Considerations}

Numerous studies have suggested a differential sex effect of glucocorticoids on neonatal outcomes $(11,12,35)$ and on surfactant phospholipid composition $(28,29,36)$. Furthermore, antenatal corticosteroids have been shown to influence maturation of the antioxidant defense system, particularly in preterm females who showed greater antioxidant enzyme activity and less oxidative stress than preterm males (37). Importantly, pro-oxidant status can alter surfactant composition and influence lung vascular reactivity, which may predispose preterm infants to poor respiratory adaptation after birth (38). Hence, it would be of interest to compare the surfactant phospholipid profile of preterm males and females with regard to antenatal glucocorticoid administration; however, in the present study only $2 / 89$ infants did not receive any antenatal corticosteroid treatment. Furthermore, chorioamnionitis and mode of delivery may influence surfactant production $(39,40)$. Further studies are required to relate differences in the surfactant phospholipid profile of preterm males and females to neonatal respiratory outcomes. It is important to note that, although gastric aspirate is likely to contain pulmonary surfactant soon after birth, not all phospholipids detected in the samples may be attributable to pulmonary surfactant, and the method of phospholipid detection can be sensitive to contamination of samples (e.g., with blood, saliva, or nasal secretions) (32).

\section{CONCLUSIONS}

Our study shows that the phospholipid composition of gastric aspirate from preterm males differs from that of preterm females and this is dependent on the gestational age at birth. We conclude that there are likely to be periods of increased vulnerability to respiratory distress in preterm males compared with females, in particular at lower gestational ages at birth.

\section{SUPPLEMENTARY MATERIAL}

Supplementary material is linked to the online version of the paper at http://www.nature.com/pr

\section{ACKNOWLEDGMENTS}

We acknowledge the assistance of PJ Meikle, JM Weir, and NA Mellett of the Metabolomics Laboratory, Baker IDI Heart and Diabetes Institute, Melbourne, Australia, in the phospholipid analysis. We also thank the nursing staff at The Royal Women's Hospital for help with gastric aspirate collection.

\section{STATEMENT OF FINANCIAL SUPPORT}

This work was supported by a Program Grant from the National Health and Medical Research Council of Australia (ID 606789).

Disclosure: The authors declare no conflict of interest.

\section{REFERENCES}

1. Binet ME, Bujold E, Lefebvre F, Tremblay Y, Piedboeuf B, Canadian Neonatal Network. Role of gender in morbidity and mortality of extremely premature neonates. Am J Perinatol 2012;29:159-66.

2. Chen SJ, Vohr BR, Oh W. Effects of birth order, gender, and intrauterine growth retardation on the outcome of very low birth weight in twins. J Pediatr 1993;123:132-6.

3. Perelman RH, Palta M, Kirby R, Farrell PM. Discordance between male and female deaths due to the respiratory distress syndrome. Pediatrics 1986;78:238-44.

4. Stevenson DK, Verter J, Fanaroff AA, et al. Sex differences in outcomes of very low birthweight infants: the newborn male disadvantage. Arch Dis Child Fetal Neonatal Ed 2000;83:F182-5.

5. Elsmen E, Hansen Pupp I, Hellstrom-Westas L. Preterm male infants need more initial respiratory and circulatory support than female infants. Acta Paediatr 2004;93:529-33.

6. Peacock JL, Marston L, Marlow N, Calvert SA, Greenough A. Neonatal and infant outcome in boys and girls born very prematurely. Pediatr Res 2012;71:305-10.

7. Thomas MR, Marston L, Rafferty GF, et al. Respiratory function of very prematurely born infants at follow up: influence of sex. Arch Dis Child Fetal Neonatal Ed 2006;91:F197-201.

8. Vento M, Cubells E, Escobar JJ, et al. Oxygen saturation after birth in preterm infants treated with continuous positive airway pressure and air: assessment of gender differences and comparison with a published nomogram. Arch Dis Child Fetal Neonatal Ed 2013;98:F228-32.

9. Anadkat JS, Kuzniewicz MW, Chaudhari BP, Cole FS, Hamvas A. Increased risk for respiratory distress among white, male, late preterm and term infants. J Perinatol 2012;32:780-5.

10. Levit O, Jiang Y, Bizzarro MJ, et al. The genetic susceptibility to respiratory distress syndrome. Pediatr Res 2009;66:693-7. 
11. Papageorgiou AN, Colle E, Farri-Kostopoulos E, Gelfand MM. Incidence of respiratory distress syndrome following antenatal betamethasone: role of sex, type of delivery, and prolonged rupture of membranes. Pediatrics 1981;67:614-7.

12. Weitzel HK, Lorenz U, Kipper B. Clinical aspects of antenatal glucocorticoid treatment for prevention of neonatal respiratory distress syndrome. J Perinat Med 1987;15:441-6.

13. Greenough A, Limb E, Marston L, Marlow N, Calvert S, Peacock J. Risk factors for respiratory morbidity in infancy after very premature birth. Arch Dis Child Fetal Neonatal Ed 2005;90:F320-3.

14. Adamson IY, King GM. Sex-related differences in cellular composition and surfactant synthesis of developing fetal rat lungs. Am Rev Respir Dis 1984;129:130-4.

15. Warburton D, Parton L, Buckley S, Cosico L, Saluna T. Beta-receptors and surface active material flux in fetal lamb lung: female advantage. J Appl Physiol (1985) 1987;63:828-33.

16. Fleisher B, Kulovich MV, Hallman M, Gluck L. Lung profile: sex differences in normal pregnancy. Obstet Gynecol 1985;66:327-0.

17. Torday JS, Nielsen HC, Fencl Mde M, Avery ME. Sex differences in fetal lung maturation. Am Rev Respir Dis 1981;123:205-8.

18. Nielsen HC, Torday JS. Sex differences in fetal rabbit pulmonary surfactant production. Pediatr Res 1981;15:1245-7.

19. Ishak N, Hanita T, Sozo F, Maritz G, Harding R, De Matteo R. Sex differences in cardiorespiratory transition and surfactant composition following preterm birth in sheep. Am J Physiol Regul Integr Comp Physiol 2012;303:R778-89.

20. Veldhuizen R, Nag K, Orgeig S, Possmayer F. The role of lipids in pulmonary surfactant. Biochim Biophys Acta 1998;1408:90-108.

21. Bhatia R, Morley CJ, Argus B, Tingay DG, Donath S, Davis PG. The stable microbubble test for determining continuous positive airway pressure (CPAP) success in very preterm infants receiving nasal CPAP from birth. Neonatology 2013;104:188-93.

22. Verder H, Ebbesen F, Fenger-Grøn J, et al. Early surfactant guided by lamellar body counts on gastric aspirate in very preterm infants. Neonatology 2013;104:116-22.

23. Sozo F, Vela M, Stokes V, et al. Effects of prenatal ethanol exposure on the lungs of postnatal lambs. Am J Physiol Lung Cell Mol Physiol 2011;300:L139-47.

24. Nielsen HC. Androgen receptors influence the production of pulmonary surfactant in the testicular feminization mouse fetus. J Clin Invest 1985;76:177-81.

25. Ishak N, Sozo F, Harding R, De Matteo R. Does lung development differ in male and female fetuses? Exp Lung Res 2014;40:30-9.
26. De Matteo R, Ishak N, Hanita T, Harding R, Sozo F. Respiratory adaptation and surfactant composition of unanesthetized male and female lambs differ for up to $8 \mathrm{~h}$ after preterm birth. Pediatr Res 2016;79:13-21.

27. Perelman RH, Engle MJ, Palta M, Kemnitz JW, Farrell PM. Fetal lung development in male and female nonhuman primates. Pediatr Res 1986; 20:987-1.

28. Freese WB, Hallman M. The effect of betamethasone and fetal sex on the synthesis and maturation of lung surfactant phospholipids in rabbits. Biochim Biophys Acta 1983;750:47-59.

29. Willet KE, Jobe AH, Ikegami M, et al. Postnatal lung function after prenatal steroid treatment in sheep: effect of gender. Pediatr Res 1997;42:885-92.

30. Orgeig S, Morrison JL, Sullivan LC, Daniels CB. The development of the pulmonary surfactant system. In: Harding R, Pinkerton KE, eds. The Lung: Development, Aging and the Environment. London, UK: Academic Press, 2015: 183-209.

31. Keough KM, Parsons CS, Tweeddale MG. Interactions between plasma proteins and pulmonary surfactant: pulsating bubble studies. Can J Physiol Pharmacol 1989;67:663-8.

32. Brown LM, Duck-Chong CG. Methods of evaluating fetal lung maturity. Crit Rev Clin Lab Sci 1982;16:85-159.

33. Hallman M, Kulovich M, Kirkpatrick E, Sugarman RG, Gluck L. Phosphatidylinositol and phosphatidylglycerol in amniotic fluid: indices of lung maturity. Am J Obstet Gynecol 1976;125:613-7.

34. Holm BA, Keicher L, Liu MY, Sokolowski J, Enhorning G. Inhibition of pulmonary surfactant function by phospholipases. J Appl Physiol (1985) 1991;71:317-21.

35. Roberge S, Lacasse Y, Tapp S, et al. Role of fetal sex in the outcome of antenatal glucocorticoid treatment to prevent respiratory distress syndrome: systematic review and meta-analysis. J Obstet Gynaecol Can 2011;33:216-6.

36. Torday JS. Androgens delay human fetal lung maturation in vitro. Endocrinology 1990;126:3240-4.

37. Vento M, Aguar M, Escobar J, et al. Antenatal steroids and antioxidant enzyme activity in preterm infants: influence of gender and timing. Antioxid Redox Signal 2009;11:2945-55.

38. Enomoto M, Gosal K, Cubells E, et al. Sex-dependent changes in the pulmonary vasoconstriction potential of newborn rats following shortterm oxygen exposure. Pediatr Res 2012;72:468-78.

39. Kunzmann S, Collins JJ, Kuypers E, Kramer BW. Thrown off balance: the effect of antenatal inflammation on the developing lung and immune system. Am J Obstet Gynecol 2013;208:429-37.

40. Callen P, Goldsworthy S, Graves L, Harvey D, Mellows H, Parkinson C. Mode of delivery and the lecithin/sphingomyelin ratio. Br J Obstet Gynaecol 1979;86:965-8. 